\title{
Effect of the coefficient of restitution and friction in the granular Leidenfrost effect in the absence of gravity. A numerical study
}

\author{
Daniel Schiochet Nasato ${ }^{1, *}$ and Heiko Briesen ${ }^{1, * *}$ \\ ${ }^{1}$ Chair of Process Systems Engineering, TUM School of Life Sciences Weihenstephan, Technical University of Munich \\ Gregor-Mendel-Straße 4, 85354 Freising, Germany
}

\begin{abstract}
In this study the granular Leidenfrost effect in the absence of gravity is investigated numerically by means of the discrete element method. Apart from identifying the phenomena, a parametric study to quantify the influence of the coefficient of restitution and friction in the packing fraction of the granular media is carried on numerically. Surprisingly, both the coefficient of restitution and the coefficient of friction exhibit an influence of the same magnitude in the packing fraction of the granular system, which has not been reported in experiments and simulation of granular Leidenfrost regime under gravity or microgravity conditions.
\end{abstract}

\section{Introduction}

Driven granular materials exhibits different behavior depending on the filling and forcing conditions of the system [1], and may be classified into granular gases, granular liquids or granular solids, according to the volume fraction of the system and the kinetic energy of the particles [2]. They may exhibit many fluid-like phenomena such as undulations, wave patterns and convection rolls [3]. Furthermore, granular gas and granular solids may coexist under certain circumstances [4]. The term granular Leidenfrost effect was first used by Eshuis et al. [4] to define a region of dense particles supported by a region of dilute fast particles (granular gas). The granular Leidenfrost can be understood as analogous to the original Leidenfrost effect [5], which describes the effect of a water droplet hovering over a hot plate, supported by a gas region. This same effect has also been employed in combination to vaporizable soft solids to power sustainable bouncing, in the so called elastic Leidenfrost effect [6].

Recently, [7] published the first experimental evidence of the existence of the granular Leidenfrost effect in microgravity, through experiments performed during a parabolic flight campaign. Reports of numerical studies of the aforementioned phenomena in the absence of gravity were not found by the authors. Howerever, there is an interesting numerical study of the influence of different parameters in the clustering transition of a vibration-driven granular gas system reported in [2]. In that study, the walls are vibrated off-phase, and the influence of friction and restitution in the phase transition is investigated. Here, a numerical investigation of the granular Leidenfrost effect in the absence of gravity is proposed. A box is shaken vertically (in-phase), and results are corroborated to those reported

\footnotetext{
*e-mail: daniel.nasato@tum.de

**e-mail: heiko.briesen@tum.de
}

A video is available at https://doi.org/10.48448/ptm7-q895 by [7]. The influence of the coefficient of restitution and the friction in the packing fraction of the granular media is investigated. Surprisingly, both parameters demonstrated to have an influence of similar magnitude in the packing fraction of the granular system here studied.

\section{Materials and Methods}

\subsection{Simulation method}

For the simulations in this paper the discrete element method (DEM) was employed by means of the software LIGGGHTS (Version 3.8.0) [8]. In DEM, the change in particles' position and velocity is calculated by integrating Newton's equation of motion. When two particles " $i$ " and " $j$ " with radius $R_{i}$ and $R_{j}$, at positions $\vec{r}_{i}$ and $\vec{r}_{j}$ are in contact, they interact and are allowed to slightly overlap. This overlap $\xi$ can be written in the normal direction as:

$$
\xi_{n}=\left(R_{i}+R_{j}-\left|\vec{r}_{i}-\vec{r}_{j}\right|\right) \hat{e}_{n}
$$

with the unit vector $\hat{e}_{n}$ obtained from:

$$
\hat{e}_{n}=\frac{\vec{r}_{i}-\vec{r}_{j}}{\left|\vec{r}_{i}-\vec{r}_{j}\right|}
$$

The contact force $\vec{F}$ is divided into a normal and a tangential component. The normal component of the force is divided into an elastic and a dissipative term, written in the form

$$
\vec{F}_{n}=\left(\vec{F} \cdot \hat{e}_{n}\right)=k_{n} \xi_{n}+c_{n} \dot{\xi}_{n}
$$

The normal force parameters, $k_{n}$ and $c_{n}$, are obtained from Hertz theory [9] in the form:

$$
k_{n}=\frac{4}{3} Y^{*} \sqrt{R^{*} \xi_{n}}
$$




$$
c_{n}=-\beta \sqrt{5 m^{*} k_{n}}
$$

where $Y^{*}$ is the equivalent Young's modulus obtained from $1 / Y^{*}=\left(1-v_{i}^{2}\right) / Y_{i}+\left(1-v_{j}^{2}\right) / Y_{j}, R^{*}$ is the equivalent radius computed from $1 / R^{*}=1 / R_{i}+1 / R_{j}$ and $m^{*}$ the equivalent mass calculated from $1 / m^{*}=1 / m_{i}+1 / m_{j}$. The parame$\operatorname{ter} \beta$ is determined from the (velocity-independent) coefficient of restitution $e$ in the form:

$$
\beta=\frac{\ln (e)}{\sqrt{\ln ^{2}(e)+\pi^{2}}}
$$

The tangential force, $\vec{F}_{t}$, leads to a torque applying to particles " $i$ " and " $j$ ", and is therefore connected to their rotational movement. The coefficient of friction $\mu_{s}$ is the upper limit of the tangential force through the Coulomb criterion $\vec{F}_{t}=\mu_{s} \vec{F}_{n}$. A simplified version of Mindlin and Deresiewicz [10] applies for the parameters in the tangential direction, $k_{t}$ and $c_{t}$. See $[11,12]$ for a detailed treatment of tangential overlap and velocity. Parameters $k_{n}, c_{n}$, $k_{t}, c_{t}$ are described in $[13,14]$.

Simulations are performed using a box with dimensions $5 \times 5 \times 5 \mathrm{~cm}$. The box is filled with particles up to a packing fraction of 0.35 . The surface of the box is discretized into a triangular mesh prior to be loaded into LIGGGHTS, and the particle-wall interactions follow the same contact law of the particle-particle interactions.

To ensure a homogeneous filling of the box, particles are inserted with $25 \%$ of their original radius, and are allowed to grow their size while inside the box until they reach their final size $(1 \mathrm{~mm})$. Afterwards, particles have their velocities set to a random value, following a Gaussian distribution with average value 0 and variance $0.1 \mathrm{~m} / \mathrm{s}$ in each direction. In total, 83557 monodisperse particles are used in each simulation. The box is shaken vertically following a sinusoidal vibration with a frequency of $25 \mathrm{~Hz}$ and an amplitude of $2 \mathrm{~mm}$ (peak-to-peak) in case 1 . In case 2 the frequency is increased to $35 \mathrm{~Hz}$ and the amplitude reduced to $1 \mathrm{~mm}$. In both cases, the coefficient of restitution is varied from 0.1 to 0.9 .

A third case (case 3 ) using the same setup of case 1 was simulated, but this time the coefficient of restitution was set to 0.3 , which is consistent with experimental measurements provided in [15], and the static friction was varied from 0.1 to 0.9 . The same coefficient of restitution and friction are used for particle-particle and particle-wall interactions. For all the cases, a Young's modulus of $5 \times 10^{7}$ was used. The box and the initial condition for the simulations are shown in Fig. 1. The parameters used in the simulations are shown in Table 1.

The virtual experiments were performed for $10 \mathrm{sec}-$ onds. Data was collected in the interval of 2 points per vibration and the results presented here are averaged over the last 50 data points collected.

\section{Results}

A comparison of packing fraction with experimental measurements for frequency $25 \mathrm{~Hz}$ and amplitude $2 \mathrm{~mm}$ (case 1) is shown in Fig. 2. A qualitatively agreement with
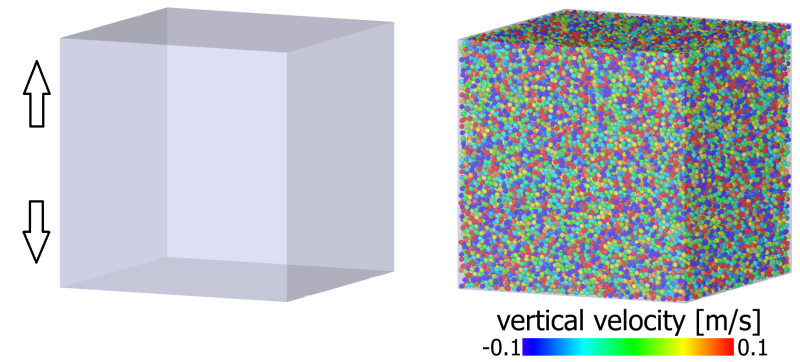

Figure 1. Numerical simulation setup. Left: box used for the simulations with the arrows indicating the vibration direction. Right: Box filled to a packing fraction of 0.35. Particles are colored by their initial vertical velocity distribution.

Table 1. Parameters used in the different simulated cases. Frequency is given in Hertz and amplitude in $\mathrm{mm}$.

\begin{tabular}{|c|c|c|c|c|}
\hline & freq & amp & coeff. of rest. & coeff. of fric. \\
\hline case 1 & 25 & 2 & 0.1 to 0.9 & 0.7 \\
\hline case 2 & 35 & 1 & 0.1 to 0.9 & 0.7 \\
\hline case 3 & 25 & 2 & 0.3 & 0.1 to 0.9 \\
\hline
\end{tabular}

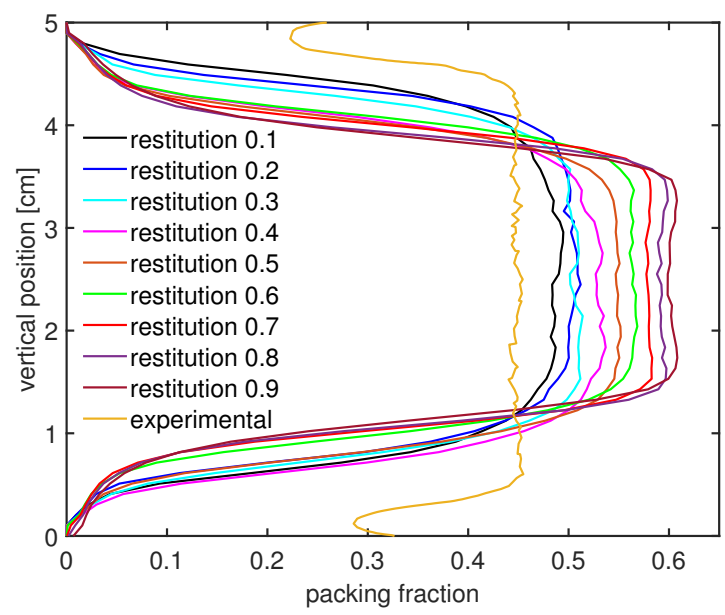

Figure 2. Numerical simulation using a frequency of $25 \mathrm{~Hz}$ and amplitude of $2 \mathrm{~mm}$. The coefficient of restitution was varied from 0.1 to 0.9 . The corresponding experimental result is also shown in the graph.

experiments was found. A quantitative agreement is not expected, as experiments were conducted in microgravity and the simulations here proposed are performed in the absence of gravity.

In Fig. 4 simulation results for case 1 using coefficient of restitution 0.1 and 0.9 are portrayed. Particles are colored by its instantaneous vertical velocity and the images are shown correspond to the system after 10 seconds of vibration. There is a clear difference in the size of dense region (slowly moving particles, colored in green), which reflects the differences in the packing fraction.

In Fig. 3 the packing fraction values for case 2 (frequency of $35 \mathrm{~Hz}$ and amplitude of $1 \mathrm{~mm}$ ) are compared to experimental measurements performed in microgravity. 


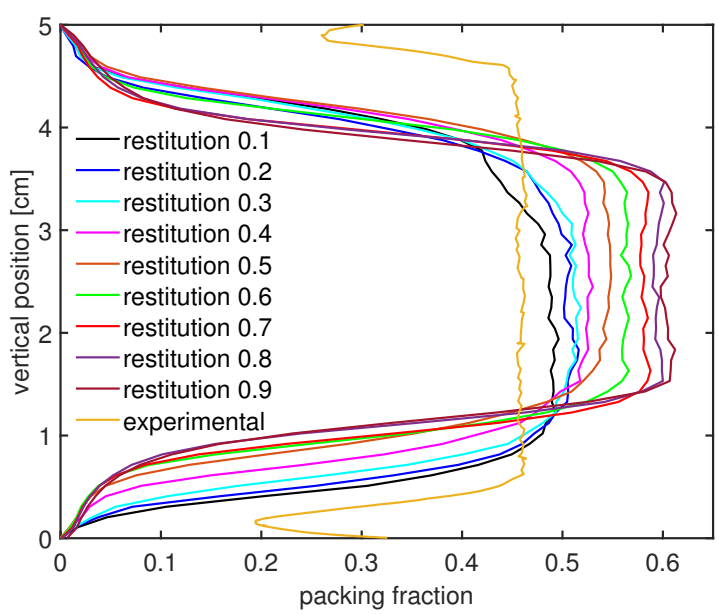

Figure 3. Numerical simulation using a frequency of $35 \mathrm{~Hz}$ and amplitude of $1 \mathrm{~mm}$. The coefficient of restitution was varied from 0.1 to 0.9 in the simulations. The corresponding experimental measurement in microgravity is shown in the graph.

As depicted in Fig. 2, a strong dependency of the packing fraction with the coefficient of restitution could be identified. By modifying the frequency and amplitude as in Fig. 3, the same dependence of the packing fraction with respect to the restitution could be noticed.

In case 3 the frequency was set to $25 \mathrm{~Hz}$, amplitude to $2 \mathrm{~mm}$, the coefficient of restitution to 0.3 [15], and the coefficient of friction was varied. The simulated cases and corresponding experimental measurements in microgravity are shown in Fig. 5. The coefficient of friction can be observed to have a large influence in the packing fraction of the granular media, being this influence in the same order of magnitude of that from the coefficient of restitution.

In Fig. 6 simulation results using a coefficient of friction of 0.1 and 0.9 (case 3) are presented. Particles are colored by its instantaneous vertical velocity and the images are shown correspond to the system after 10 seconds of vibration. Similar to cases 1 and 2, there is a clear difference in the size of the clusters formed, which reflects the differences in the packing fraction.

\section{Conclusion}

The granular Leidenfrost effect is characterized by a a dilute region of fast moving particles supporting a region of dense particles. This effect has been demonstrated by under the effect of gravity [4], and recently demonstrated experimentally in the condition of microgravity [7]. Evidence of the granular Leidenfrost effect in the absence of gravity was demonstrated here by means of numerical simulations for the first time. Furthermore, the influence of the coefficient of restitution and friction in the packing fraction is demonstrated.

Here an interesting dependence of the packing fraction with respect to the coefficient of restitution, as well as with the coefficient of friction, was identified. An influence of the coefficient of restitution in the solid fraction
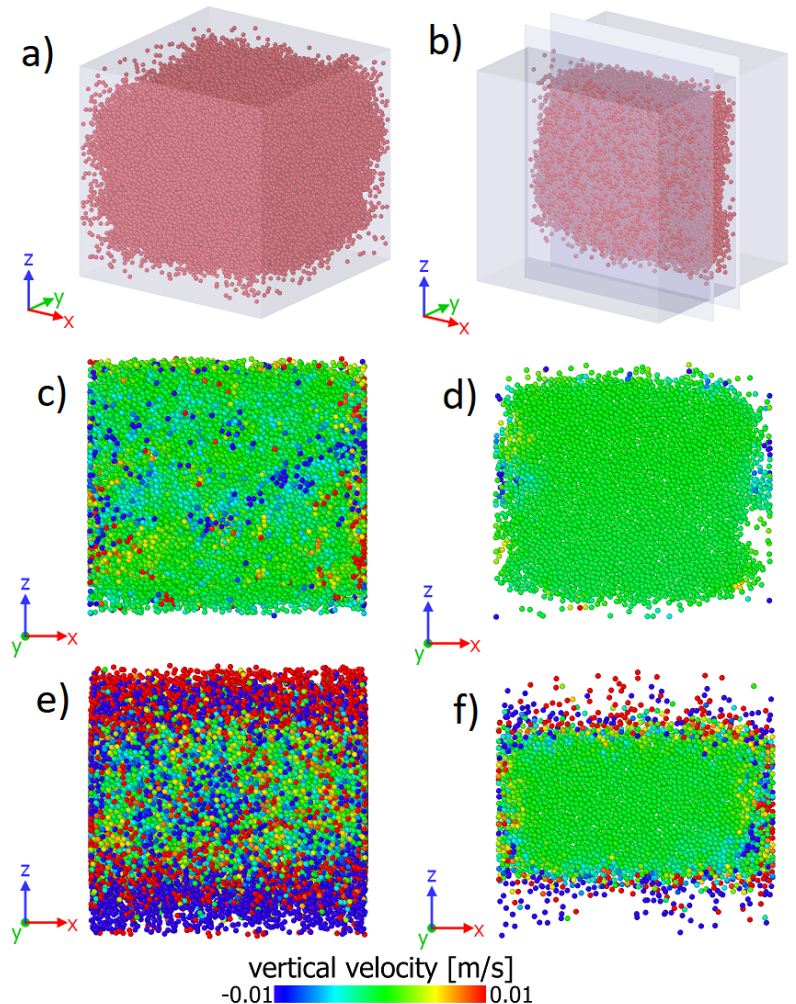

Figure 4. Simulation results of case 1 using a coefficient of restitution of 0.1 and 0.9. In a) is depicted the full simulation box and the respective slice through its middle plane is shown in b). In c) is the full case using a coefficient of restitution of 0.1 , and in d) its slice taken from the full case. In e) is the full case using a coefficient of restitution of 0.9 , and in $f$ ) is the corresponding slice taken from the full case. In a) and b) the view is in perspective, while the other images are in front view. See the coordinate tripod for reference.

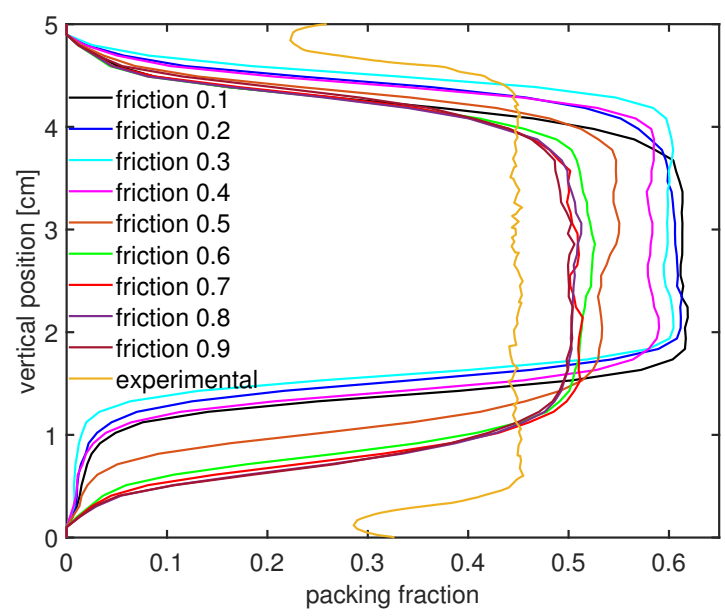

Figure 5. Numerical simulation using a frequency of $25 \mathrm{~Hz}$ and amplitude of $1 \mathrm{~mm}$. Coefficient of friction was varied from 0.1 to 0.9 . Corresponding experimental result is also shown in the graph. 
a)

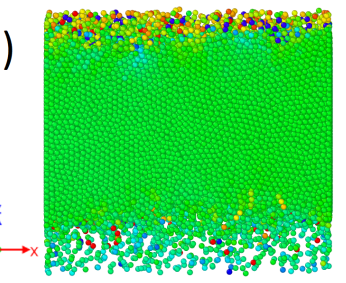

c)

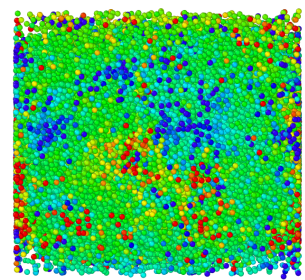

\section{b)}

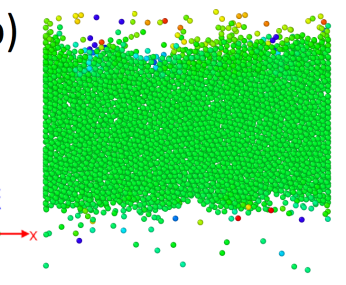

d)

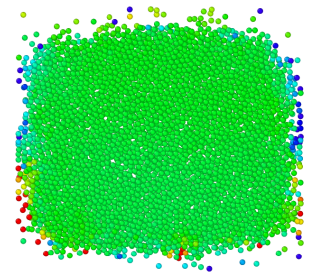

vertical velocity $[\mathrm{m} / \mathrm{s}]$

$0.01[0.01$

Figure 6. Numerical simulation results of case 3 using a coefficient of friction of 0.1 and 0.9 . In a) is the full case using a coefficient of friction of 0.1 , and in b) is a slice taken from the full case. In c) is the full case using a coefficient of friction of 0.9 , and in d) is the corresponding slice taken from the full case. See Fig. 4 a) and b) for correct spatial location of the slice plan.

was already expected, as studies of vertically shaken granular matter under gravity take the inelasticity parameter $\epsilon=\left(1-e^{2}\right)$ into consideration [3] when phase diagrams are discussed. The coefficient of restitution have shown a direct influence in the packing density. However, no direct influence in the formation or absence of the granular Leidenfrost effect could be identified.

Surprisingly, the coefficient of friction seems to have a direct influence in the packing fraction, in a very similar fashion as the coefficient of restitution. The maximum packing fraction increased from approximately 0.5 to 0.6 when the coefficient of restitution was increased from 0.1 to 0.9 . In a similar pattern, the maximum packing fraction increased from approximately 0.5 to 0.6 when the coefficient of friction was reduced from 0.9 to 0.1 . Nonetheless, the coefficient of friction is not mentioned to have a direct influence in the packing fraction of vertically vibrated granular media under gravity [3] or in low-gravity environment [16], and is therefore firstly identified in this study.

The numerical results greed qualitatively with measurements performed under microgravity. The experiments, as mentioned in [7], may have spurious gravity acceleration due to some imperfections in the parabolic flight which may have a large influence in the solid fraction distribution inside the vibrated container. Additionally, regions near the walls (below 0.5 and above $4.5 \mathrm{~cm}$ ) may incorporate interference of the vibrating container's walls, which would generate artificially darken these regions and explain the larger packing fraction measured through Xray near the walls. This assumption is, however, not discussed in the experiments. Another possible source of error is the velocity-independent coefficient of restitution model adopted in DEM simulations. This, however, does not invalidate the study here demonstrated, as the whole range of coefficient of restitution was investigated in this parametric study and vertical velocities do not exceed 0.9 $\mathrm{m} / \mathrm{s}$ in all cases simulated.

Further studies should identify the sources of the deviations from experimental results, as well as investigate the influence of different operational parameters, e.g. the global solid fraction in the cell, frequency and amplitude. Nevertheless, this study inspired the authors at thinking on this experiment as a suitable to investigate material properties. With advances in X-ray tomography, similar experiments to the one proposed by [7] could be performed, e.g., in a drop tower [17], as an alternative to investigate elastic and frictional properties of granular materials, as the behavior seems to be quite sensitive to both.

\section{References}

[1] P. G. de Gennes, Rev. Mod. Phys. 57, 827 (1985)

[2] Q.L. Wu, M-Y. Hou, L. Yang, W. Wang, G.H. Yang, K.W. Tao, L.W. Chen, S. Zhang, Chin. Phys. B 29, 054502 (2020)

[3] P. Eshuis, K. van der Weele, D. van der Meer, D. Lohse, Phys. Fluids. 19, 123301 (2007)

[4] P. Eshuis, K. van der Weele, D. van der Meer, D. Lohse, Phys. Rev. Lett. 95, 258001 (2005)

[5] J.G. Leidenfrost, Int. J. Heat Mass Transfer 9, 1153 (1966)

[6] S.R. Waitukaitis, A. Zuiderwijk, A. Souslov, C. Coulais, M. van Hecke, Nature Physics 13, 1095-1099 (2017)

[7] H. Torres Menéndez, A. Sack, T. Pöschel, Granular Matter 22, 67 (2020)

[8] C. Kloss, C. Goniva, A. Hager, S. Amberger, S. Pirker, Prog. Comput. Fluid Dy. 12, 264 (2012)

[9] H. Hertz, J. f. reine u. angewandte Math., 92, 156-171 (1882)

[10] R.D. Mindlin, H. Deresiewicz, Trans. ASME J. Appl. Mech., 20, 327-344 (1953)

[11] S. Luding, Granul. Matter 10, 235-246 (2008)

[12] T. Pöschel, D.S. Nasato, E.J.R. Parteli, J.A.C. Gallas, P. Müller, Am. J. Phys. 87 (4), 255-263 (2019)

[13] A. Di Renzo, F.P. Di Maio, Chem. Eng. Sci. 59, 525541 (2004)

[14] Y. Tsuji, T. Tanaka, T. Ishida, Powder Technol. 71 (3), 239-250 (1992)

[15] C. Mangwandi, Y.S. Cheong, M.J. Adams, M.J. Hounslow, A.D. Salman, Chem. Eng. Sci. 62, 437-450 (2007)

[16] M. Noirhomme, A. Cazaubiel, A. Darras, E. Falcon, D. Fischer, Y. Garrabos, C. Lecoutre-Chabot, S. Merminod, E. Opsomer, F. Palencia, J. Schockmel, R. Stannarius, N. Vandewalle, EPL 123, 14003 (2018)

[17] N. Callens, J. Ventura-Traveset, E.Z. GarciaAndrade, C. Gómez-Calero, J.J.W.A. van Loon, V. Pletser, E. Kufner, J. Krause, R. Lindner, F. Gai, C. Eigenbrod, ESA parabolic flight, drop tower and centrifuge hands-on activities, in Proceedings of the 61st International Astronautical Congress, IAC 2010, 27 September - 1 October 2010, Prague, Czech Republic (2010) 